\title{
Cardiovascular development: structure and molecular mechanism
}

\author{
Yuji Nakajima
}

Published online: 1 April 2009

(C) Japanese Association of Anatomists 2009

For more than 100 years, anatomists, physiologists, biologists, pathologists, oncologists, and cardiologists have been fascinated by the study of cardiovascular development. The cardiovascular system, which consists of many cell types, such as endothelial cells, myocardial cells, and smooth muscle cells, to name only a few, develops from the mesoderm and the ectomesenchyme. The circulatory system is established and functions at the earliest stage of development as it is essential for normal embryonic development. In the clinical field of pediatric cardiology, there are a number of congenital heart defects that are commonly diagnosed in about $1 \%$ of live births. In other clinical fields, angiogenesis and lymphangiogenesis are critical processes because they influence the invasiveness of cancer as well as the survival of transplanted grafts. We often see cardiovascular anomalies in human bodies in the dissection lab as well as in genetically engineered mutant animals. To date, many Japanese scientists have investigated and made landmark discoveries in this field. In 1978, Professor Atsuyoshi Takao (1925-2006), who was the first pediatric cardiologist in Japan, initiated international symposia on cardiovascular development, which developed into a long-lasting series. The proceedings of these symposia illustrate the rapid and remarkable progress that has been made in this field.

A satellite symposium entitled "Mechanisms regulating cardiovascular development" was held during the 112th Annual Meeting of the Japanese Association of

\footnotetext{
Y. Nakajima $(\bowtie)$

Department of Anatomy and Cell Biology, Graduate School of Medicine,

Osaka City University, 1-4-3 Asahimachi,

Abenoku, Osaka 545-8585, Japan

e-mail: yuji@med.osaka-cu.ac.jp
}

Anatomists, 27-29 March 2007, Osaka. Experts in the field were invited to actively participate at this symposium, which was aimed at improving our understanding of the structure and molecular mechanism regulating the development of the cardiovascular system. In this Special Issue, we summarize the talks given at the symposium: the first three papers describe early heart development; the reviews that follow describe vascular development; and the final paper investigates lymphangiogenesis.

During vertebrate embryogenesis, the heart starts to beat at the early neurula stage; therefore, functional cardiomyocytes are formed soon after gastrulation. Dr. Y. Nakajima (Osaka City University Graduate School of Medicine) reviews early cardiomyocyte development, including the cellular movement of cardiomyocyte progenitors and the signaling mechanisms that regulate cardiomyogenesis from the blastula to gastrula stages. Heart progenitors reside in the posterior lateral region of the epiblast during the blastula stage, subsequently moving to the anterior half of the primitive streak and then migrating anterolaterally to form the anterior lateral plate mesoderm that contains the precardiac mesoderm, which has been recently termed the first heart field. A two-step signaling system that consists of Nodal, fibroblast growth factor (FGF)8, and bone morphogenetic protein (BMP)-antagonists at the blastula stage and BMP, FGF, and Wnt-antagonists at the gastrula stage is involved in specification/determination and terminal differentiation.

During morphogenetic remodeling from a single heart tube to a four-chambered heart, endocardial cushion tissue, which is derived from the endocardial epithelium via the epithelial-mesenchymal transition (EMT), plays a central role, as it gives rise to the anlagen of valves and septa. In his presentation, Dr. T. Yamagishi (Saitama Medical University) describes his research which involved the use 
of a three-dimensional collagen gel culture model of the endocardial EMT to identify BMP as one of the myocardial-derived inductive signals that initiate the endocardial EMT. He then shows that the signaling mediated by BMP and transforming growth factor-beta (TGF $\beta$ ) plays a central role in the formation of the valvulo-septal endocardial cushion tissue. Dr. Yamagishi also reviews the downstream signaling of TGF $\beta$ and BMP and suggests that several type I receptors, such as ALK1 and -5 for TGF $\beta$ and ALK 2, -3, and -6 for BMP interact with each other to regulate the endocardial EMT.

Heart structure develops from multiple cell sources, such as the first heart field in the lateral plate mesoderm and the second heart field in the splanchnic/pharyngeal mesoderm, cardiac neural crest, and proepicardial organ. Consequently, abnormal development of the cardiogenic progenitors causes congenital heart defects. Dr. H. Yamagishi (Keio University School of Medicine) reviews cardiovascular development from its multiple embryonic origins and focuses on normal and abnormal development of the recently re-discovered second heart field, which has been found to give rise to the heart outflow tract and right ventricle. Tbx1, which encodes a T-box-containing transcription factor, is the major candidate gene for the $22 \mathrm{q} 11.2$ deletion syndrome. Using a genetic approach in mice, Dr. Yamagishi first identified that Fox transcription factors upregulate the expression of Tbx1, FGF8, and FGF10 in the second heart field. He then showed that a reduction in the level of Tbx1 in the second heart field causes hypoplasia of the outflow tract and right ventricle, resulting in the conotruncal malformations seen in 22q11.2 deletion syndrome. It has recently been reported that downregulation of FGF8 in the second heart field causes abnormal cardiomyocytes in the outflow tract, which then fail to express TGF $\beta /$ BMP and the extracellular matrix, causing hypoplastic cushions and septation defects to form in the outflow tract. The discovery of the second heart field provides new insights into cardiac morphogenesis and congenital heart defects.

The vascular system is the first organ that develops prior to other organogenesis as it provides oxygen and nutrients. During the initial phase of vascular development, endothelial precursors establish a capillary plexus via vasculogenesis and are subsequently remodeled into organized vessels. Dr. M. Hirashima (Kobe University Graduate School of Medicine) discusses the mechanisms regulating the differentiation of endothelial cells, acquisition of arterial endothelial identity, and patterning of the vasculature. First, he summarizes the origin of endothelial cells: hemangioblasts derived from the splanchnic mesoderm contribute to vasculogenesis in visceral organs and angioblasts from somites in the somatopleura. He shows that vascular endothelial growth factor (VEGF) and Notch signaling coordinate with each other and play a major role in vascular development and patterning. VEGF-A, with its three distinct receptors, controls endothelial proliferation, migration, and survival. Notch signaling induces arterial endothelial differentiation during initial vasculogenesis, while it negatively regulates VEGF-induced angiogenesis. In addition to embryogenesis, vasculogenesis/angiogenesis is closely related to pathological angiogenesis as well as to regenerative medicine.

The lymphatic system is important for fluid homeostasis, fat absorption, and the immune system as well as several pathological conditions. Despite the importance of the lymphatic system, the process of lymphangiogenesis is poorly understood because of the lack of specific markers for lymph vessels. Using a genetically engineered zebrafish model to study lymphangiogenesis, Dr. S. Isogai (Iwate Medical University School of Medicine), presents research data showing that zebrafish have a lymph vascular system similar to those of other vertebrates, including mammals, and that lymph vessels, such as the thoracic duct in zebrafish, originate from venous endothelial cells. He also summarizes the history of lymphology and reviews the phylogeny and ontogeny of the lymph system in vertebrates.

The authors of the articles appearing in this Special Issue wish to thank the Editor in Chief of Anatomical Science International for extending the opportunity to present their work. We hope that the issue will encourage young scientists to work in this field and that it provides useful information towards improving our understanding of how our cardiovascular system has developed. 